\title{
Distinct genetic basis for root responses to lipo-chitooligosaccharide signal molecules from
}

distinct microbial origins

Maxime Bonhomme ${ }^{1 *}$, Sandra Bensmihen ${ }^{2 * \dagger}$, Olivier André ${ }^{1}$, Emilie Amblard ${ }^{1}$, Magali Garcia $^{1}$, Fabienne Maillet ${ }^{2}$, Virginie Puech-Pagès ${ }^{1}$, Clare Gough ${ }^{2}$, Sébastien Fort ${ }^{3}$, Sylvain Cottaz $^{3}$, Guillaume Bécard ${ }^{1}$, Christophe Jacquet ${ }^{1, \dagger}$

${ }^{1}$ Laboratoire de Recherche en Sciences Végétales, Université de Toulouse, CNRS, UPS, Castanet-Tolosan, France.

${ }^{2}$ LIPME, Université de Toulouse, INRAE, CNRS, 31326 Castanet-Tolosan, France.

${ }^{3}$ Univ. Grenoble Alpes, CNRS, CERMAV, 38000 Grenoble, France.

* These authors contributed equally to this work

$\uparrow$ authors for correspondence: Sandra.bensmihen@inrae.fr, phone +33561285463

and jacquet@1rsv.ups-tlse.fr, phone : +33 (0)5 34323814

other e-mail addresses: Maxime Bonhomme: Bonhomme@lrsv.ups-tlse.fr; Olivier André: Andreo@Desangosse.com; Emilie Amblard: emilie.amblard@1rsv.ups-tlse.fr; Magali Garcia: magali.garcia@laposte.net; Fabienne Maillet: fabienne.maillet@inrae.fr; Virginie PuechPages: puech@1rsv.ups-tlse.fr; Clare Gough: clare.gough@inrae.fr; Sébastien Fort: Sebastien.Fort@cermav.cnrs.fr; Sylvain Cottaz: sylvain.cottaz@cermav.cnrs.fr; Guillaume Bécard: becard@1rsv.ups-tlse.fr

\section{ORCID}

Maxime BONHOMME : https://orcid.org/0000-0002-1210-4777

Sandra BENSMIHEN : https://orcid.org/0000-0003-1351-7220

Christophe JACQUET : https://orcid.org/0000-0002-7377-5638

Clare GOUGH : https://orcid.org/0000-0001-5844-7217

Sébastien FORT: https://orcid.org/0000-0002-6133-9900

Guillaume BECARD: https://orcid.org/0000-0002-5085-7577 


\section{Abstract}

Lipo-chitooligosaccharides (LCOs) were originally found as symbiotic signals called Nod Factors (Nod-LCOs) controlling nodulation of legumes by rhizobia. More recently LCOs were also found in symbiotic fungi and, more surprisingly, very widely in the kingdom fungi including in saprophytic and pathogenic fungi. The LCO-V(C18:1, Fuc/MeFuc), hereafter called Fung-LCOs, are the LCO structures most commonly found in fungi. This raises the question of how legume plants, such as Medicago truncatula, can discriminate between Nod-LCOs and these Fung-LCOs. To address this question, we performed a Genome Wide Association Study on 173 natural accessions of Medicago truncatula, using a root branching phenotype and a newly developed local score approach. Both Nod- and Fung-LCOs stimulated root branching in most accessions but the root responses to these two types of LCO molecules were not correlated. Also, heritability of root response was higher for Nod-LCOs than for Fung-LCOs. We identified 123 loci for Nod-LCO and 71 for Fung-LCO responses, but only one was common. This suggests that Nod- and Fung-LCOs both control root branching but use different molecular mechanisms. The tighter genetic constraint of the root response to Fung-LCOs possibly reflects the ancestral origin of the biological activity of these molecules.

Keywords: GWAS, lateral root development, lipo-chitooligosaccharides, Medicago truncatula, Nod Factors.

Abbreviations: CSSP: Common Symbiosis Signalling Pathway; Fuc/MeFuc : Fucosylated/ Methyl fucosylated; GWAS: Genome Wide Association Study; LCOs: Lipochitooligosaccharides; LRF: Lateral Root Formation; RLS: Rhizobium Legume Symbiosis, AM: arbuscular mycorrhiza. 


\section{Introduction}

Lipo-chitooligosaccharides (LCOs) constitute a family of chitin oligomers substituted on their non-reducing end with an acyl chain, and further substituted with a variety of additional functional groups. LCOs were originally found, 30 years ago, to be symbiotic signals, called Nod factors, produced by rhizobia to trigger the nodulation process in legumes (Dénarié et al., 1996). This discovery was the starting point for a series of work that gradually brought to light the symbiotic signaling pathway required for rhizobial infection and nodulation in legumes. Nod-LCO perception shows a high host specificity that relies on precise LCO substitutions (Dénarié et al., 1996), and specific LCO structures are perceived by Lysin-motif Receptor Like Kinases (LysM-RLKs) in host plants (Bensmihen et al., 2011; Radutoiu et al., 2007). The activation of the downstream signaling pathway, now called the Common Symbiosis Signalling Pathway (CSSP), was also found to be necessary for root colonization by Arbuscular Mycorrhizal (AM) fungi (Catoira et al., 2000). Furthermore, it was subsequently discovered that LCOs with high structural similarity to Nod factors are also produced by AM fungi (so called Myc-LCOs, Fig. S1) (Maillet et al., 2011). Without genetic proof that these molecules are essential for mycorrhization, but since they activate the CSSP as well as symbiotic gene expression changes in host plants, they are considered, together with their oligosaccharidic precursors (COs), as key mycorrhizal signals (Camps et al., 2015; Genre et al., 2013; Gough and Cullimore, 2011; Sun et al., 2015). This was supported by the recent finding in Solanum lycopersicum, that the receptor protein SILYK10 binds Myc-LCOs and is involved in the AM symbiosis (Girardin et al., 2019). Also recently, Cope et al. showed both that the CSSP is used for establishment of the ectomycorrhizal symbiosis between Laccaria bicolor and poplar, and that L. bicolor can produce LCOs with similar structures to Nod factors (Cope et al., 2019). Possibly linked to their roles as symbiotic signals, LCOs can interfere with immunity-related signaling in legumes (Rey et al., 2019) and suppress innate immune responses, even in the nonmycorrhizal plant Arabidopsis thaliana (Liang et al., 2013). How LCOs dampen legume immunity is still unclear as they can also induce defense gene expression (Nakagawa et al., 2011). Another property of LCOs is their ability to modify root architecture by stimulating Lateral Root Formation (LRF). The stimulation of LRF appears to be a general response, observed in legume species such as Medicago truncatula treated with Nod Factors or MycLCOs (Maillet et al., 2011; Olah et al., 2005), but also in the monocots rice and Brachypodium distachyon (Buendia et al., 2019; Sun et al., 2015). In Medicago and Brachypodium, the effect of LCO stimulation of LRF occurs at early stages of root development and is seen on seedlings grown in vitro (Herrbach et al., 2017; Buendia et al., 2019). Other positive effects of LCOs on 
soybean or maize root development are reported (Souleimanov et al., 2002; Tanaka et al., 2015). So, up to this point in our knowledge, LCOs were considered as signal molecules produced by a variety of symbiotic microorganisms and with several effects on plants, including activation of the CSSP, regulation of immune responses and stimulation of root development. However, very recently, a new LCO chapter was opened when Rush et al. (Rush et al., 2020) discovered both that AM fungi produce a wider range of LCOs than previously described, and that LCOs are not exclusive to symbiotic microorganisms. Indeed, they constitute a family of molecules commonly produced by a very large number of fungi, in all clades of the fungi kingdom. As such, LCOs produced by fungi will be thereafter referred to as "Fung-LCOs" (Supplementary Fig. S1 available at $J X B$ online). Like previously characterized LCOs, FungLCOs consist of oligomers of 3- 5 residues of $\mathrm{N}$-acetyl glucosamine acylated with fatty acid chains of various length, saturated or not, and are decorated with acetyl, $N$ methyl, carbamoyl, fucosyl, fucosyl sulfate, methyl fucosyl or sulfate groups. They can be found in phytopathogenic fungi, but also in saprophytes and opportunistic human pathogens, i.e. in nonsymbiotic fungi or in fungi that do not interact with plants. The results of Rush et al. suggest that Fung-LCOs are conserved molecules in fungi that can regulate endogenous developmental processes such as spore germination, hyphal branching, or dimorphic switching. The fact that LCO-producing fungi of all kinds are abundantly present in the close environment of plant roots raises many new questions.

Focusing on the plant side, some of these questions are: are these Fung- LCO structures able to trigger similar root responses, especially the LRF stimulation previously observed in response to Nod- and Myc-LCOs? If so, are legumes nevertheless able to differentiate these Fung-LCOs from the Nod-LCOs? To address these questions, we used a natural variability approach to compare root growth responses to Fung-LCOs and Nod-LCOs, using the model plant Medicago truncatula. As a legume, this plant must distinguish between Nod factors specifically produced by its rhizobial symbiont, Sinorhizobium meliloti, and Fung-LCOs molecules commonly produced by a vast number of rhizospheric fungi. We carried out two Genome-Wide Association Studies (GWAS) within a collection of 173 accessions of M. truncatula (Bonhomme et al., 2014), whose seedlings were either treated with cognate Nod-LCOs, mainly LCO-IV(C16:2, Ac, S), or with the Fung-LCOs, LCO-V(C18:1, Fuc/MeFuc) (Rush et al., 2020). By doing so, we could compare root responses to Nod- and Fung-LCOs in a way that is not possible solely using the reference A17 genotype, and we could uncover specific genetic determinants underlying these root responses. Our results shed light on how legumes can cope with structurally related signals emitted by distinct rhizospheric microbes. 


\section{Materials and Methods}

\section{Production of lipo-chitooligosaccharide molecules}

The Fung-LCOs used here were LCO-V(C18:1, Fuc/MeFuc, Fig. S1) synthesized by metabolically engineered Escherichia coli as described in (Chambon et al., 2015; Ohsten Rasmussen et al., 2004; Samain et al., 1999; Samain et al., 1997), the fucosyl and methylfucosyl substitutions on the reducing end were obtained as described in (Djordjevic et al., 2014). They were chosen as they are the most representative of the fungal LCOs (Rush et al., 2020). Sinorhizobium meliloti Nod factors, named thereafter "Nod-LCOs" [mainly LCO-IV(C16:2, Ac, S)] were extracted from $S$. meliloti culture supernatants by butanol extraction, and purified by high-performance liquid chromatography (HPLC) on a semi-preparative C18 reverse phase column, as described in (Roche et al., 1991b). Nod-LCO and Fung-LCO structures (Fig. S1) were verified by mass spectrometry as described in (Cope et al., 2019).

\section{Plant material, experimental design and root phenotyping}

A collection of 173 M. truncatula accessions (http://www.medicagohapmap.org), provided by the INRAE Medicago truncatula Stock Center (Montpellier, France; www1.montpellier.inra.fr/BRC-MTR/), was used for phenotyping experiments. These accessions are representative of the overall genetic diversity of $M$. truncatula and belong to the CC192 core collection (Ronfort et al., 2006). GWAS for various phenotypic traits have already been performed using this collection (Bonhomme et al., 2014; Bonhomme et al., 2019; Kang et al., 2015; Stanton-Geddes et al., 2013; Yoder et al., 2014).

M. truncatula seeds were scarified with sulfuric acid, sterilized in bleach (2.5\%) for four minutes, washed in sterile water, and transferred on sterile agar plates for 2.5 days in the dark at $15^{\circ} \mathrm{C}$ to synchronize germination. Seedlings were then grown in vitro on square Petri dishes $(12 \times 12 \mathrm{~cm})$ under $16 \mathrm{~h}$ light and $8 \mathrm{~h}$ dark at $22^{\circ} \mathrm{C}$, with a $70^{\circ}$ angle inclination, on modified $\mathrm{M}$ medium, as previously used for other M. truncatula LRF assays and described in (Bonhomme et al., 2014) and (Herrbach et al., 2017). This medium contained either (i) the "Nod" treatment in which Nod-LCOs were incorporated at a final concentration of $10^{-8} \mathrm{M}$, (ii) the "Fung" treatment in which Fung-LCOs, 10 times less water soluble than the sulfated Nod-LCOs, were incorporated at a concentration of $10^{-7} \mathrm{M}$ (Ohsten Rasmussen et al., 2004), or (iii) two control (CTRL) conditions corresponding to a 1000x dilution in acetonitrile 50\% (CTRL-Fung) or to a 100 000X dilution in water (CTRL-Nod). Different solvents were used for the two types of LCOs as Nod-LCOs are sulfated and more soluble in water than the methyl-fucosylated Fung- 
LCOs (see Fig. S2 and S3). Each accession of M. truncatula was phenotyped in two independent biological repeats, with 15 seedlings per repeat ( 3 plates of 5 seedlings per plate), for each treatment (Nod, Fung, CTRL-Nod, CTRL-Fung).

For each treatment, the lateral root number (LR) of each seedling was followed at four time points of plant development: 5, 8, 11 and 15 days after seedling transfer on the experimental media. In addition, the primary root length (RL) was measured 5- and 11-days post treatment in order to calculate the lateral root density (LRD, i.e. the ratio of the lateral root number over the primary root length of each plant). All these measurements were carried out using the image analysis software Image J, using scans of plates (see Fig. S5-S6). In order to summarize the kinetics of lateral root number appearance over the four time points, we calculated for each plant the Area Under the Lateral Root Progress Curve -AULRPC- (Fig. S4) using the R statistical package “agricolae". Overall, nine phenotypic variables were recorded for each plant and for each treatment: LR_5d, LR_8d, LR_11d, LR_15d, RL_5d, RL_11d, LRD_5d, LRD_11d and AULRPC.

\section{Statistical modeling of phenotypic data}

For the Nod and Fung treatments separately, as well as for the control of each treatment (i.e. mock treated plants of the Nod- or Fung-LCOs experiments), adjusted means of each accession (coefficients) were estimated for each of the nine phenotypic variables by fitting the following linear model with fixed effects: $\mathrm{y}_{i j k}=\operatorname{accession}_{i}+$ repeat $_{j}+\varepsilon_{i j k}$, where $\mathrm{y}_{i j k}$ is the phenotypic value of the $k$ th plant of the $j$ th repeat for the $i$ th accession. Since variation in the root system development naturally occurred within and among accessions both in control and Nod/Fung-treated plants, for LR, RL, LRD and AULRPC variables, an additional variable of induction/repression of the root system development was estimated for each accession by subtracting the coefficient value under treatment with Nod- or Fung-LCOs by the coefficient value under control condition (i.e. CTRL-Nod or CTRL-Fung). GWAS was performed using these variables, referred to as "delta", estimated for each accession on Nod and Fung-LCOs treatments separately (delta_LR_5d, delta_RL_5d, delta_LRD_5d, delta_LR_8d, delta_LR_11d, delta_RL_11d, delta_LRD_11d,delta_LR_15d, delta_AULRPC).

\section{Association mapping and local score analyses of phenotypic data}

GWAS was performed on the phenotypic variables described in the previous section, based on phenotypic values for 173 accessions of M. truncatula (see Tables S1-S4 for details). We used the Mt4.0 Medicago genome and SNP version to perform GWAS (see 
http://www.medicagohapmap.org/). A set of 5,165,380 genome-wide SNPs was selected with a minor allele frequency of $5 \%$ and at least $90 \%$ of the 173 accessions scored across the $M$. truncatula collection. The statistical model used for GWAS was the mixed linear model (MLM) approach implemented in the EMMA expedited (EMMAX) software (Kang et al., 2010). The MLM is used to estimate and then test for the significance of the allelic effect at each SNP, taking into account the genetic relationships between individuals to reduce the false positive rate. Genetic relationships among accessions were estimated using a kinship matrix of pairwise genetic similarities which was based on the genome-wide proportion of alleles shared between accessions, using the whole selected SNP dataset.

The MLM first implements a variance component procedure to estimate the genetic $\left(\sigma_{\mathrm{a}}^{2}\right)$ and residual $\left(\sigma_{\mathrm{e}}^{2}\right)$ variances from the variance of the phenotypic data, by using the kinship matrix in a restricted maximum likelihood framework. Narrow-sense heritabilities (i.e. portion of the total phenotypic variation attributable to additive genetic effect, $\mathrm{h}^{2}{ }_{\mathrm{ss}}$ ) of each phenotypic variable were calculated from estimates of $\sigma^{2}$ a and $\sigma^{2}$. For each marker a Generalized Least Square $F$-test is used to estimate the effects $\beta_{\mathrm{k}}$ and test the hypothesis $\beta_{\mathrm{k}}=0$ in the following model: $y_{\mathrm{i}}=\beta_{0}+\beta_{\mathrm{k}} \mathrm{X}_{\mathrm{ik}}+\eta_{\mathrm{i}}$, with $\mathrm{X}_{\mathrm{ik}}$ the allele present in individual $i$ for the marker $k$, and $\eta_{\mathrm{i}}$ a combination of the random genetic and residual effects (Kang et al., 2010). As in previous GWAS in M. truncatula (Bonhomme et al., 2014; Rey et al., 2017), we used a genome-wide $5 \%$ significance threshold with Bonferroni correction for the number of blocks of SNPs in linkage disequilibrium (i.e. $p$-value $\leq 10^{-6}$ ), to identify significant associations following the $F$ test on the estimated allele effect size at each SNP.

In order to detect small-effect QTL that would not pass the $10^{-6}$ significance threshold, we performed a local score approach (Aoun et al., 2020; Bonhomme et al., 2019; Fariello et $a l ., 2017)$ on SNP $p$-values. The local score is a cumulative score that takes advantage of local linkage disequilibrium (LD) among SNPs. This score, defined as the maximum of the Lindley process over a SNP sequence (i.e. a chromosome), as well as its significance threshold were calculated based on EMMAX $p$-values, using a tuning parameter value of $\xi=3$, as suggested by simulation results (Bonhomme et al., 2019). R scripts used to compute the local score and significance thresholds are available at https://forge-dga.jouy.inra.fr/projects/localscore/documents.

\section{Results}

Natural variation in the stimulation of lateral root formation by Fung- and Nod-LCOs in Medicago truncatula 
Growth of the 173 accessions of $M$. truncatula in the presence of Fung-LCOs or NodLCOs led to $67 \%$ and $87 \%$ of them with delta_AULRPC values above 0 , respectively. This indicates a global trend of LCO stimulation of lateral root formation (LRF), especially with Nod-LCOs (Fig. 1A, B). This trend appeared early in the experiment since LRF was stimulated in $72 \%$ and $83 \%$ of the accessions 5 days following Fung-LCO and Nod-LCO treatments, respectively (Table 1). Interestingly, while certain lines showed a significant stimulation, some lines showed significant repression of LRF upon Nod or Fung-LCO treatment (see Fig. 1 and Fig. S9-S12). Among these lines, the reference genotype A17 was strongly stimulated by NodLCOs over the time course, but not by Fung-LCOs (Fig. 1A, B). The R108 genotype was not included in our GWAS panel because it is not part of the CC192 HapMap collection we used. However, as it is commonly used for reverse genetics approach, we also tested its root response to Fung-LCOs (Supplementary Fig. S5 and S6). A higher branching phenotype than that of A17 in control conditions was observed, and no significant LRF or primary root length responses could be detected for R108 upon Fung-LCO treatment (Fig.S5 and S6).

Since LRF stimulation showed substantial variation across the $M$. truncatula collection, we estimated the heritability, namely the proportion of phenotypic variation observed that was due to genetic variation in the collection (Table 1). In response to Fung-LCOs, the heritability was relatively low $\left(\mathrm{h}^{2}{ }_{\mathrm{ss}} \leq 0.16\right)$ for phenotypic variables quantifying variation in $L R$ number and density, and showed a clear tendency to increase over time $\left(\mathrm{h}^{2}{ }_{\mathrm{ss}}=0.16\right.$ for LR number at 15 days post treatment and $\mathrm{h}^{2}{ }_{\mathrm{ss}}=0.15$ for LR density at 11 days). In contrast, in response to Nod-LCOs the heritability of variation in LR number and density was strong at early times (i.e. 0.66 and 0.75 at 5 days post treatment, respectively) and decreased over time but remained relatively high (i.e. $>0.22$ and 0.35 , respectively). Interestingly, variation of primary root length in response to Fung- and Nod-LCOs was also observed, with a more frequent stimulation effect. Its heritability was stronger for Nod-LCOs at 11 days $\left(\mathrm{h}^{2}{ }_{\mathrm{ss}}=0.36\right.$, Table 1$)$. In the case of treatment with Nod-LCOs, these results indicate that variation in LRF stimulation, but also in primary root length stimulation, was largely due to genetic variation in the collection, especially at early steps, showing the importance of natural variation in the genetic control of LRF and primary root length stimulation by Nod-LCOs in M. truncatula. In the case of treatment with Fung-LCOs, the results may indicate a higher sensitivity of LRF to environmental - here experimental - conditions. However, the strong level of LRF stimulation as well as the low heritability at early steps $\left(0 \leq \mathrm{h}^{2}{ }_{\mathrm{ss}} \leq 0.06\right.$, see Table 1$)$ support the hypothesis that the root response to Fung-LCOs in M. truncatula is much more genetically constrained than the root response to Nod-LCOs. 
Since Fung and Nod-LCOs show a high structural homology and both stimulated LRF in most genotypes, we tested whether accessions highly stimulated by Nod-LCOs were also highly stimulated, not stimulated or even repressed by Fung-LCOs. Interestingly, for all variables, we found no correlation between the stimulations by Fung- and Nod-LCOs, except at 5 days where we found a significant but weak positive correlation for the variation in LR number $(r=0.15, p$-value $=0.024)$. The lack of global correlation between LRF stimulation by Fung-LCOs and LRF stimulation by Nod-LCOs is illustrated in (Fig. 1C, D), with the delta_AULRPC variable that captures root development over time, and with the LR number at 5 days (delta_LR_5d) that captures early steps of root development.

Overall, these results suggest that (i) both Fung- and Nod-LCOs have the property to stimulate LRF and primary root length in a quantitative manner, and (ii) genetic variation seems more influential in the root response to Nod-LCOs than to Fung-LCOs. To better understand the genetic determinants underlying these contrasted phenotypic responses, we performed a Genome-Wide Association Study.

\section{Genetic determinants underlying quantitative variation in root responsiveness to Fung- and Nod-LCOs in Medicago truncatula}

GWAS was performed separately for Fung and Nod-LCO treatments, for each of the nine phenotypic variables measuring: (i) variation of the lateral root number (delta_LR_5d, delta_LR_8d, delta_LR_11d and delta_LR_15d), (ii) lateral root density (delta_LRD_5d and delta_LRD_11d), (iii) primary root length (delta_RL_5d, delta_RL_11d) and (iv) lateral root progress curve (delta_AULRPC) over time (5, 8, 11 and 15 days) (see Tables S1-S4). Across all phenotypic variables measured in response to Fung-LCOs and Nod-LCOs, $p$-value-based tests performed using EMMAX respectively identified 24 and 70 genomic regions or loci significant at the $p$-value threshold of $10^{-6}$. Using the local score approach, more significant candidate genomic regions were identified as associated with root response to Fung- and NodLCOs, respectively 71 and 123 loci and 1 common locus (Table S5). All the loci identified with the EMMAX approach are nested within the local score results. Identified loci contain 1 to 11 genes, corresponding to 291 possible genes in total (see Table S5). The mean number of genes per interval was $1.5 \pm 1.26 \mathrm{SD}$, indicating that most of our GWAS hits correspond to a single gene. Importantly, some loci were identified for the same treatment using several phenotyping parameters (highlighted in Table S5, in green for Fung-LCOs, orange for Nod-LCOs, blue for both). 
A global view of the genome-wide quantitative genetic bases of LRF stimulation kinetics following treatment with LCOs could be obtained by the local score analysis of the delta_AULRPC variable (Fig. 2A, B). Genetic variation involved in LRF stimulation specifically in response to Fung-LCOs mainly relied on four candidate loci; a gibberellin 2oxidase (Medtrlg086550, GA2OX) and three receptor-like kinases: a putative Feronia receptorlike kinase - Medtr6g015805-, a crinkly 4 receptor like kinase CCR4-like protein Medtr3g464080 -, and a Serine/Threonine kinase PBS1 - Medtr8g063300 - (Fig. 2A, Table S5). One major locus on chromosome 7, containing genes from the leguminosin LEED.PEED family (Trujillo et al., 2014), but also kinase encoding genes with potential carbohydratebinding properties (S-locus lectins) were specifically involved in response to Nod-LCOs (Fig. 2B, Table S5). Only one candidate genomic region involved in the response to both Fung-LCOs and Nod-LCOs was identified in this study, by the GWAS analysis of delta_AULRPC and primary root length (delta_RL_5d) phenotypic variables (Table S5). This region on chromosome 8 contains three genes among which two encode "embryonic abundant protein", annotated as BURP domain-containing protein by the new $M$. truncatula genome version Mt5 (Pecrix et al., 2018).

A more precise view of the genome-wide quantitative genetic bases of the early steps of LRF stimulation following treatment with LCOs could be obtained by the local score analysis of the delta_LRD_5d variable (Fig. 2C, D). Interestingly, this phenotypic variable showed highly contrasted heritability values between treatments with Fung- and Nod-LCOs $\left(\mathrm{h}^{2}{ }_{\mathrm{ss}}=0.06\right.$ and 0.75 , respectively; Table 1). Among 34 candidate genomic regions identified in response to Fung-LCOs, we identified four highly significant candidate genes whose predicted proteins show good homology for known functions, such as a dioxygenase (Medtr5g055800, annotated as "2OG-Fe(II) oxygenase family oxidoreductase"), an LRR receptor-like kinase (Medtr3g452970), a WRKY family transcription factor (Medtr5g091390) and a GRAS family transcription factor (Medtr4g097080) whose homolog in Arabidopsis thaliana is SHORTROOT -SHR- (Helariutta et al., 2000). Among 49 candidate genomic regions identified in response to Nod-LCOs for the delta_LRD_5d variable, we identified 4 highly significant candidate genes, among which two are predicted to encode dioxygenases (Medtr4g100590, Medtr2g068940, annotated "2OG-Fe(II) oxygenase family oxidoreductase"), one MYB transcription factor (Medtr5g081860, MYB51), and the most significant one encoding a putative membrane lipoprotein lipid attachment site-like protein (Medtr8g464760), annotated as thioredoxin-like protein in the Mt5 genome. This analysis also detected two known genes 
encoding a sugar transporter (Medtr3g098930, MtSWEET11) and a GRAS family transcription factor (Medtr8g442410, TF124) (Fig. 2D).

The local score approach for the phenotypic parameter of primary root length at 5 days identified several F-box containing genes among the main hits obtained, both for Nod-LCO and Fung-LCO treatments (Fig. S7). Among them, the F-box containing genes Medtr6g027420 and Medtr6g006730 obtained for the Fung-LCO and Nod-LCO responses, respectively, belong to the RNI-subfamily of SKP Ubiquitin ligase adaptor proteins that are involved in several hormonal responses such as strigolactone or auxin signalling (Mashiguchi et al., 2020; Sparks et al., 2013). Interestingly, a locus with a low $p$-value $\left(\leq 2.39 \times 10^{-10}\right)$ at the end of chromosome 5 corresponded to a gene (Medtr5g085250) similar to the E3 ligase COP1 that was shown to control root growth in response to shoot-perceived light in Arabidopsis (Sassi et al., 2012). Similarly, two putative auxin efflux carriers, similar to the PIN-like PILS 5 and 7 proteins from Arabidopsis (Sauer and Kleine-Vehn, 2019) were found associated with the primary root response after 5 days in response to Nod-LCOs. For the root growth response to Fung-LCOs at 5 days, we also identified the MtSWEET3c locus that was shown to transport mannose and sucrose and to be expressed in Medicago nodules or roots upon abiotic stress (Hu et al., 2019).

Thus, the GWAS hits we found for the LRF or root growth responses identified putative candidates with relevant functions for both root development and possible symbiotic roles.

\section{Global analysis of GWAS hits}

The most significant genomic regions detected by GWAS thus gave a first hint at possible mechanisms involved in root response to LCOs. However, local score analysis also highlighted minor QTL/genes and allowed us to identify several dozen of supplementary genes. To gain further insights from these data, we performed a Gene Ontology (GO) enrichment analysis using the Medicago Superviewer interface (Herrbach et al., 2017) (Fig. S8 and made a literature search using the knowledge database LEGOO (Carrère et al., 2020) (Fig.3 and Table S6).

Based on GO analysis, 71 and 134 genes identified in the Fung-LCO and Nod-LCO GWAS were classified, respectively. Both the Nod and Fung-LCO datasets were enriched in biological functions related to "other metabolic processes" ( $\mathrm{p}$-value=0.012 and $5.584 \times 10^{-3}$, respectively). The Nod-LCO data were also enriched in transcription related biological processes $\left(p-\right.$ value $\left.=2.036 \times 10^{-3}\right)$. The Fung-LCO data was, as the Nod-LCO data, enriched in transcription factor and kinase activities ("molecular function" level, $\mathrm{p}$-value $=5.005 \times 10^{-3}$ and 0.011 for Nod-LCOs and $\mathrm{p}$-value $=0.022$ or 0.021 for Fung-LCOs, respectively) (Fig. S8 A, B). 
This is in accordance with the numerous loci associated with receptor-like kinases or transcription factors (TF) found in both datasets (see Table S1). Accordingly, the Nod-LCO data showed enrichment in nuclear $\left(\mathrm{p}\right.$-value $\left.=3.792 \times 10^{-4}\right)$ and plasma membrane $(\mathrm{p}$-value $=$ $3.907 \times 10^{-5}$ ) associated "cellular component" (Fig. 3B). Many of the metabolic functions from the Nod-LCO candidates and of the genes underlying the "protein metabolism" biological process enriched with Fung-LCOs ( $p$-value $=0.027$ ) were associated with phosphorylation, so possibly also with signaling pathways. In addition, a significant proportion of loci were associated with oxido-reduction processes and cell-wall metabolism enzymes (pectin-esterases, cellulose synthase, phenylalanine ammonia-lyase-like protein). Although not specifically enriched in these datasets, we also found several hormone related genes. For instance, auxin signaling (AUX/IAA and Auxin Response Factor, ARF) and auxin transport (efflux carriers) genes were found in the Nod-LCO data whereas an ethylene receptor and an ethylene responsive TF were found in the Nod-LCO and Fung-LCO data, respectively (Table S5).

Analysis of transcriptional expression data from the literature that is accessible in the M. truncatula knowledge database (Carrère et al., 2020) retrieved genes differentially expressed in different biological conditions. Data could be obtained for 148 out of the 291 candidate genes and are summarized in Table S6. Figure 3 shows the overlap of the gene expression patterns found between the different conditions analysed. Overall, our GWAS results identified 123 out of these 148 genes (i.e 83.1\%) in symbiotic studies (nodulation or mycorrhization see "relationship_organism” column of Table S6) and 25 out of 148 (16.9\%) in transcriptomic studies with LCO treatments (yellow circle and see "relationship_molecule" column of Table S6) (Fig. 3A). However, available expression data were not restricted to these symbiotic interactions. Indeed, for the loci identified in both GWAS analysis, expression data could also be retrieved from nitrate or phosphate starvation experiments (found for 67/148 genes (green circle, Fig. 3A), eight of these 67 genes were specific to nitrate or phosphate responses, 55/67 genes were also found in symbiotic expression data) or from data obtained using M. truncatula root pathogens or defense elicitors (found for 53/148 genes (red circle): 9 of these 53 genes were specific to pathogen conditions, 40/53 genes were also found with symbiotic expression) (Figure 3A, Table S6). A closer look at the loci identified specifically in the Fung - (Fig. 3B) or Nod - (Fig. 3C) LCO data separately showed a similar proportion of genes specifically related to nutrition (green circles: 11.5\% (3/26) for Fung- and 10\% (4/40) for Nod-LCOs) or to pathogen defense (red circles, $14.3 \%$ (3/21) for Fung- and 18.75\% (6/32) for Nod-LCOs). 
To summarize, a more global view of our GWAS results highlighted enrichment in signaling and metabolic functions and identified genes that can be responsive to biotic (symbiosis but also pathogenesis) and abiotic (nutrient status) interactions.

\section{Discussion}

In this study, we asked whether a similar developmental root response triggered by lipochitooligosaccharide molecules that share similar structures could depend on similar or different molecular mechanisms in the model legume $M$. truncatula. Regulation of root development by LCOs seems to be a conserved plant response observed in legume and nonlegume plants (Buendia et al., 2019; Sun et al., 2015; Tanaka et al., 2015), raising questions about its evolutionary origin and molecular conservation. The Nod-LCO molecules we used, LCO-IV(C16:2, Ac, S), are produced by the rhizobial symbiont of M. truncatula. These LCOs can be considered as very specific symbiotic signals, with a key role in the narrow host specificity that characterizes the rhizobium legume symbiosis (RLS). The simple absence of the sulfate group on the reducing end of the Nod-LCOs renders them inactive symbiotically on Medicago (Bensmihen et al., 2011a; Roche et al., 1991a). In contrast, the Fung-LCO molecules used here, LCO-V(C18:1, Fuc/MeFuc), are not only a form of LCOs commonly found in AM fungi, but they can also be produced by pathogenic or saphrophytic fungi (Rush et al., 2020) and can thus be considered as a common, almost universal, hallmark of fungal presence. Furthermore, it is worth noticing that even Bradyrhizobia and Sinorhizobium symbionts of soybean also produce LCO-V(C18:1, Fuc/MeFuc) (D'Haeze and Holsters, 2002; Wang et al., 2018), making them also non cognate Nod-LCO signals for M. truncatula. By studying the ability of M. truncatula plants to respond to specific (Nod-LCOs) or wide-spread (Fung-LCOs) LCOs, we were thus considering a common situation encountered by plant roots in their natural environment where they can face different LCO-producing microorganisms.

Here, we have exploited the large genetic diversity among $M$. truncatula natural accessions using a GWAS approach to compare the genetic basis underlying root developmental responses. The root phenotypic traits that we used, lateral root formation and lateral root density, were chosen because in the $M$. truncatula reference accession A17 these traits are stimulated by Nod factors and by the Myc-LCOs originally detected in AM fungi (Fig. S1) (Maillet et al., 2011; Olah et al., 2005). Moreover, this root developmental response could be a way to address the activity of LCO structures produced by a wider range of fungi, and not only symbiotic ones. To address LR density, we also looked at primary root growth, a parameter 
that was not previously described as affected by Nod-LCOs in A17. Moreover, these traits are relatively easy to score, which was convenient to phenotype many accessions of $M$. truncatula. It is worth noting that, in our growth conditions, all accessions formed LRs, albeit with very different total numbers.

\section{Fung-LCO and Nod-LCO structures stimulate root development of Medicago truncatula in a quantitative way}

Our results clearly showed that the Fung-LCO molecules tested, LCO-V (C18:1, Fuc/MeFuc) can also stimulate LRF in M. truncatula. This LRF stimulation is variable among the accessions, and the trait would have been missed if we had only studied the reference accession, A17, which is poorly responsive (Fig. 1), as previously shown with Sinorhizobium fredii Nod factors, LCO-V (C18:1, MeFuc) (Olah et al., 2005). We even found accessions that showed a significant inhibition of LRF upon Nod-LCO or Fung-LCO treatments (Fig. S9- S12). Also, in contrast to what was previously observed in A17 (Olah et al., 2005), we could detect some positive effect of Nod-LCOs on primary root length, especially at later time points (11 days). The majority of accessions responded positively to Fung-LCOs for this growth parameter at both 5 and 11 days. Accordingly, we found a number of loci associated with the variation in primary root length phenotype (Table S5). Among these loci were some F-box containing genes, an E3 ligase $C O P 1$ related gene and putative auxin efflux carriers that could control root growth in response to environmental cues. This underlines the power of the natural variation approach that can detect more responsive genetic backgrounds and reveal new genetic determinants that would have passed unnoticed in forward and reverse genetic screens with classical reference accessions. Similarly, GWAS results obtained on root architecture modification of Arabidopsis thaliana upon hormonal treatments identified that the Col-0 reference accession is not the most responsive to auxin (Ristova et al., 2018).

\section{Medicago truncatula can distinguish between Fung-LCOs and Nod-LCOs}

The lack of overlap, with only one exception and for different parameters, between the loci identified in the Nod-LCO and Fung-LCO GWAS is striking. This lack of overlap is consistent with the weak correlation between the ability of one accession to respond to Nodand to Fung-LCOs (Fig. 1). Although GWAS approaches cannot detect conserved (non variable) genetic loci, comparing the overlap in genetic variation for a given trait in response to similar environmental clues is theoretically possible. For instance, in a previous work, we 
used GWAS to find loci underlying the resistance of $M$. truncatula to five strains of the oomycete pathogen Aphanomyces euteiches and identified shared and specific loci, most of which being identified thanks to the local score approach only (Bonhomme et al., 2019). The absence of common genes (except one locus) highlighted in the two GWAS, and the very different heritability values found associated with the Fung-LCO and Nod-LCO responses, indicate that $M$. truncatula clearly distinguishes these signals, although they have similar structures and cause the same root response. This can be due to specific receptors (no data is available yet concerning plant receptors for the Fung-LCOs we used) and/or to divergence in downstream signaling pathways. The latter hypothesis is consistent with the enrichment in signaling functions we observed in the GWAS genes (Fig. S8). Nod-LCO and Myc-LCO stimulation of LRF requires the CSSP in M. truncatula (Maillet et al., 2011; Olah et al., 2005). However, previous transcriptomic studies performed with Myc-LCO structures which are closer to those of Nod-LCOs from S. meliloti (Fig. S1) identified that Myc-LCO signaling can also act independently of the CSSP gene MtDMI3 (Camps et al., 2015; Czaja et al., 2012). It would be interesting to test whether the Fung-LCOs we used here require signaling from the CSSP to activate the LRF responses in $M$. truncatula. CSSP mutants are available in the $M$. truncatula A17 genetic background but this accession is poorly responsive to these new FungLCOs in our assays (see Fig. 1 and Fig. S3). In the same way, it would be difficult to test mutants in the R108 background that does not show a better root response to Fung-LCOs than A17 (Fig. S3 and S4).

\section{Genetic determinants of M. truncatula responses to Fung-LCOs and Nod-LCOs}

Cell wall, root growth and developmental signaling pathway associated loci

Only one of the genes or loci identified in the two GWAS analyses was found to be common. This region contained two genes annotated as BURP domain-containing proteins, which represent a group of proteins specific to plants (Table S5). The BURP domain was named from the four members of the group initially identified, BNM2, USP, RD22, and PG1beta and is commonly found in plant cell wall proteins (Hattori et al., 1998; Wang et al., 2015). Cellwall related functions, like-cell-wall remodeling, could be linked to root growth promotion activities of the LCO molecules, and additionally might be related to the root hair deformation capacities of LCOs (Esseling et al., 2003). One "BURP" gene from this locus (Medtr8g046000) was previously described as down-regulated by Nod-LCOs in the root epidermis (4h after 10${ }^{8} \mathrm{M}$ Nod-LCO treatment) (Jardinaud et al., 2016), downregulated in nodules at 4 and $10 \mathrm{dpi}$, 
compared to roots (El Yahyaoui et al., 2004) and upregulated in roots mycorrhized with Rhizophagus irregularis at 28 dpi compared to non-mycorrhizal control roots (Hogekamp et al., 2011) (see Table S6).

In the Fung-LCO GWAS, we found some signaling genes that could have a role in LRF. These are the receptor like kinase (RLK) CRINKLY 4 (CCR4) (Medtr3g464080), and a GRAS TF (Medtr4g097080) related to the SHORTROOT gene of Arabidopsis, known to control root development (De Smet et al., 2008; Helariutta et al., 2000). MtCCR4 has not been characterized in M. truncatula yet but Medtr4g097080 corresponds to MtSHR2, recently found to act redundantly with MtSHRI to control nodule number and root length in M. truncatula (Dong et $a l ., 2020)$. This suggests that most of the loci we identified could mainly be required for lateral root development, and would only play a role in symbiosis if recruited for the formation of the symbiotic lateral root organ, the nodule. Among the putative RLK genes detected in the FungLCO GWAS, one could encode a Feronia-like RLK (Medtr6g015805). Interestingly, this protein regulates root growth of A. thaliana (Haruta et al., 2014) but also plant immune signaling by sensing cell-wall integrity (Stegmann et al., 2017), two biological processes also regulated by LCOs. Similarly, we identified several receptor-like cytosolic kinases (RLCKs), also known as PBS1-like kinases, from the subfamily VII in the Nod-LCO data. Some genes from this subfamily are involved in PAMP-triggered immunity (PTI), including chitin responses in A. thaliana (Rao et al., 2018).

\section{Phytohormone associated loci}

Relatively few hormone-related genes were identified in the two GWAS and they were all different. The ethylene-related genes Medtrlg069985 and Medtrlg073840 were found in Fung-LCO and Nod-LCO GWAS, respectively. A gibberellin-related GA2 oxidase gene (Medtrlg086550) and a few auxin transporter genes (Medtr5g024530, Medtr5g024560 and Medtr5g024580) were found in the Fung-LCO and Nod-LCO GWAS, respectively. GA2 oxidase is predicted to be a catabolic enzyme that degrades gibberellins (GA) (Yamaguchi, 2008). In M. truncatula, in contrast to Arabidopsis, GAs are negative regulators of LRF (Fonouni-Farde et al., 2019). GAs are also negative regulators of nodulation and mycorrhization (Bensmihen, 2015; Foo et al., 2013) so down regulation of the GA content could stimulate LRF, nodulation and mycorrhization. Interestingly, all the auxin-related functions were found in the Nod-LCO GWAS only. This could be related to the tight developmental links between LR formation and nodule organogenesis and their common need for auxin accumulation in M. truncatula (Schiessl et al., 2019; Soyano et al., 2019). 


\section{Endosymbiosis associated loci}

Several other loci we identified could also be related to symbiosis. When comparing with previous transcriptomic studies, we found 123 genes (78 for Nod-LCOs, 44 for FungLCOs and one found in both studies) expressed during symbiotic processes (nodulation or mycorrhization, Table S6). This represents an important overlap (123/291=42\%, although we did not retrieve expression data for all 291 loci) probably linked to the role of these molecules as pre-symbiotic or symbiotic signals to prepare or control specific symbiotic events. We could even find some very specific LEED...PEED loci that are specifically expressed in nodules (Trujillo et al., 2014) in the A17 genetic background. Along the same line, MtSWEET11 (found for the difference in LRD at 5 days with Nod-LCOs, Table S1) was previously shown to be expressed in infected root hairs, and more specifically in infection threads and symbiosomes during nodulation in M. truncatula. However, knock out of this gene did not impair RLS, possibly due to genetic redundancy (Kryvoruchko et al., 2016). This illustrates the interest of GWAS to identify genes without redundancy issues that can mask the detection of a phenotype by reverse genetics. Some genes identified in our Nod-LCO GWAS were also found in a previous GWAS of nodulation. For example, Medtrl g064090/Medtrlte064120 (annotated as a phenylalanine ammonia-lyase-like protein / Copia-like polyprotein/retrotransposon) and Medtr2g019990 (annotated as a Serine/Threonine-kinase PBS1-like protein) were previously found by Stanton-Geddes and colleagues as associated with nodule numbers in the lower part of the root (Stanton-Geddes et al., 2013). Two other loci Medtr3g034160 (galactose oxidase) and Medtr5g085100 (AP2 domain class transcription factor) were respectively found as associated with nodule numbers in the upper part of the root and with strain occupancy in the lower part of the root (Stanton-Geddes et al., 2013).

We did not find any known CSSP or LysM-RLK genes among our loci detected by GWAS. This is somehow expected as constrained natural variability on these essential symbiotic genes due to selective processes was often found in previous nucleotide polymorphism analyses (De Mita et al., 2006; De Mita et al., 2007; Grillo et al., 2016) and in previous GWAS studies performed on nodulation phenotypes (Stanton-Geddes et al., 2013). This also suggests that these genes are not major determinants of natural variability in root developmental responses to LCOs, although some LysM-RLK genetic variants likely account for rhizobia host-specificity (Sulima et al., 2017; Sulima et al., 2019). In a more general manner, it is worth noting that our GWAS approach would not detect conserved non variable core elements of root development or symbiotic signalling, especially given that all our 
accessions are able to nodulate or to form LRs, showing there is no variability for these two traits. So we cannot rule out that stimulation of LRF by LCOs involves some common components we could not identified here.

\section{Evolutionary origins of Medicago truncatula responses to Fung-LCOs and Nod-LCOs}

Our GWAS results also raise interesting questions on the evolutionary origin of the root growth stimulation abilities of LCOs. Indeed, the two different LCO structures (from different microbial origins) triggered LRF stimulation on a high number of Medicago accessions. The low heritability of plant responses to Fung-LCOs (with a maximum of 0.16 for the difference in LR number at 15 days), compared to that of plant responses to Nod-LCOs (with a maximum of 0.75 for lateral root density at 5 days) is not due to a lack of activity of the Fung-LCOs since $67 \%$ to $76 \%$ of the accessions did show an increase in root growth response upon treatment with these LCOs. This rather suggests that the genetic determinants of the Fung-LCO responses are more "fixed" (i.e. less variable) than those of the Nod-LCO responses. Despite this quite low heritability, we could still identify relevant loci. This was possible thanks to the local score approach that, as previously shown with the pathogen Aphanomyces euteiches, enables detection of loci with 5 to $10 \%$ heritability (Bonhomme et al., 2019). The low genetic variability of responses to these widespread Fung-LCO structures is likely linked to their very ancient appearance in the fungi kingdom (Rush et al., 2020), and suggests that the ancient function(s) of these LCOs were non symbiotic. Ancient LCO functions could be LRF stimulation or the regulation of immunity in plants (Feng et al., 2019; Liang et al., 2013; Limpens et al., 2015), a function that may have predated the mycorrhizal symbiosis and has not been lost in Arabidopsis (Liang et al., 2014). LCOs could also be involved in other aspects of plant biology, yet to be discovered.

\section{Conclusion}

Our study provides novel insights into the molecular basis underlying root developmental responses to LCOs from different biological origins. Further reverse genetic studies are required to prove the actual importance of these novel loci. For the moment, such approaches are challenging since both A17 and R108 genetic backgrounds- where most of the reverse genetic tools are available- were poorly responsive to the Fung-LCO treatment. So any future reverse genetic approaches should be undertaken in novel accession backgrounds, at least for Fung-LCOs. A precise investigation of the different allelic variants of these loci would 
represent an additional approach but would require deeper sequencing of the accessions showing extreme phenotypes. The very low overlap in the loci identified in response to these two LCO treatments does not rule out the existence of some conserved core regulatory genes, such as CSSP genes, in controlling these root developmental responses. Nevertheless, our data showing distinct genetic architectures of the root responses to these specific and non-specific LCO signals strongly suggests that their recognition has had distinct evolutionary histories, and provide evidence for an ancestral role of LCOs for stimulation of root growth.

\section{Acknowledgments}

This work was part of a program funded by the French Agence Nationale de la Recherche (ANR-14-CE18-0008, "NICE CROPS"). The authors thank the bioinformatics platform Toulouse Midi-Pyrenees (Genotoul). Thanks to V. Regard for help with formatting of TableS6. Mass spectrometry analyses were done with the support from the ICT-Mass Spectrometry and MetaToul-AgromiX facilities and from the MetaboHUB-ANR-11-INBS-0010 network. S.F. and S.C. received technical support of ICMG (FR 2607) mass spectrometry platform and partial financial support from the LABEX ARCANE and CBH-EUR-GS (ANR-17-EURE-0003), Glyco@Alps (ANR-15-IDEX-02), and PolyNat Carnot Institut (ANR-16-CARN-0025-01). This work was performed in the LRSV and LIPME (Toulouse, France), parts of the "Laboratoire d'Excellence" (LABEX) entitled TULIP (ANR-10-LABX-4).

\section{Author contributions}

MB, SB: analyzed the data; MB, SB, CG, GB, CJ: wrote the manuscript; MB, GB, CG, CJ: designed the experiments; OA, EA, MG, FM, VPP: performed the experiments; SF, SC: synthesized the Fung-LCO molecules.

\section{Data availability statement}

All data supporting the findings of this study are available within the paper and within its supplementary materials published online. 


\section{References}

Aoun N, Desaint H, Boyrie L, Bonhomme M, Deslandes L, Berthomé R, Roux F. 2020. A complex network of additive and epistatic quantitative trait loci underlies natural variation of Arabidopsis thaliana quantitative disease resistance to Ralstonia solanacearum under heat stress. Mol Plant Pathol 21, 1405-1420.

Bensmihen S. 2015. Hormonal Control of Lateral Root and Nodule Development in Legumes. Plants (Basel) 4, 523-547.

Bensmihen S, de Billy F, Gough C. 2011. Contribution of NFP LysM Domains to the Recognition of Nod Factors during the Medicago truncatula/Sinorhizobium meliloti Symbiosis. PLoS One 6, e26114. Bonhomme M, André O, Badis Y, Ronfort J, Burgarella C, Chantret N, Prosperi JM, Briskine R, Mudge J, Debéllé F, Navier H, Miteul H, Hajri A, Baranger A, Tiffin P, Dumas B, Pilet-Nayel ML, Young ND, Jacquet C. 2014. High-density genome-wide association mapping implicates an F-box encoding gene in Medicago truncatula resistance to Aphanomyces euteiches. New Phytol 201, 13281342.

Bonhomme M, Fariello MI, Navier H, Hajri A, Badis Y, Miteul H, Samac DA, Dumas B, Baranger A, Jacquet C, Pilet-Nayel ML. 2019. A local score approach improves GWAS resolution and detects minor QTL: application to Medicago truncatula quantitative disease resistance to multiple Aphanomyces euteiches isolates. Heredity (Edinb) 123, 517-531.

Buendia L, Maillet F, O'Connor D, van de-Kerkhove Q, Danoun S, Gough C, Lefebvre B, Bensmihen S. 2019. Lipo-chitooligosaccharides promote lateral root formation and modify auxin homeostasis in Brachypodium distachyon. New Phytol 221, 2190-2202.

Camps C, Jardinaud MF, Rengel D, Carrère S, Hervé C, Debellé F, Gamas P, Bensmihen S, Gough C. 2015. Combined genetic and transcriptomic analysis reveals three major signalling pathways activated by Myc-LCOs in Medicago truncatula. New Phytol 208, 224-240.

Carrère SB, Verdenaud M, Gough C, Gouzy JRM, Gamas P. 2020. LeGOO: An Expertized Knowledge Database for the Model Legume Medicago truncatula. Plant Cell Physiol 61, 203-211.

Catoira R, Galera C, de Billy F, Penmetsa RV, Journet EP, Maillet F, Rosenberg C, Cook D, Gough C, Dénarié J. 2000. Four genes of Medicago truncatula controlling components of a nod factor transduction pathway. Plant Cell 12, 1647-1666.

Chambon R, Despras G, Brossay A, Vauzeilles B, Urban D, Beau JM, Armand S, Cottaz S, Fort S.

2015. Efficient chemoenzymatic synthesis of lipo-chitin oligosaccharides as plant growth promoters. Green Chem. 17, 3923-3930.

Cope KR, Bascaules A, Irving TB, Venkateshwaran M, Maeda J, Garcia K, Rush TA, Ma C, Labbé J, Jawdy S, Steigerwald E, Setzke J, Fung E, Schnell KG, Wang Y, Schlief N, Bücking H, Strauss SH, Maillet F, Jargeat P, Bécard G, Puech-Pagès V, Ané JM. 2019. The Ectomycorrhizal Fungus Laccaria bicolor Produces Lipochitooligosaccharides and Uses the Common Symbiosis Pathway to Colonize Populus Roots. Plant Cell 31, 2386-2410.

Czaja LF, Hogekamp C, Lamm P, Maillet F, Martinez EA, Samain E, Dénarié J, Küster H, Hohnjec N.

2012. Transcriptional responses toward diffusible signals from symbiotic microbes reveal MtNFP- and MtDMI3-dependent reprogramming of host gene expression by arbuscular mycorrhizal fungal lipochitooligosaccharides. Plant Physiol 159, 1671-1685.

D'Haeze W, Holsters M. 2002. Nod factor structures, responses, and perception during initiation of nodule development. Glycobiology 12, 79R-105R.

De Mita S, Santoni S, Hochu I, Ronfort J, Bataillon T. 2006. Molecular evolution and positive selection of the symbiotic gene NORK in Medicago truncatula. J Mol Evol 62, 234-244.

De Mita S, Santoni S, Ronfort J, Bataillon T. 2007. Adaptive evolution of the symbiotic gene NORK is not correlated with shifts of rhizobial specificity in the genus Medicago. Bmc Evolutionary Biology 7. De Smet I, Vassileva V, De Rybel B, Levesque MP, Grunewald W, Van Damme D, Van Noorden G, Naudts M, Van Isterdael G, De Clercq R, Wang JY, Meuli N, Vanneste S, Friml J, Hilson P, Jurgens G, Ingram GC, Inze D, Benfey PN, Beeckman T. 2008. Receptor-like kinase ACR4 restricts formative cell divisions in the Arabidopsis root. Science 322, 594-597. 
Djordjevic MA, Bezos A, Susanti, Marmuse L, Driguez H, Samain E, Vauzeilles B, Beau JM, Kordbacheh F, Rolfe BG, Schwörer R, Daines AM, Gresshoff PM, Parish CR. 2014. Lipo-chitin oligosaccharides, plant symbiosis signalling molecules that modulate mammalian angiogenesis in vitro. PLoS One 9, e112635.

Dong W, Zhu Y, Chang H, Wang C, Yang J, Shi J, Gao J, Yang W, Lan L, Wang Y, Zhang X, Dai H, Miao Y, Xu L, He Z, Song C, Wu S, Wang D, Yu N, Wang E. 2020. An SHR-SCR module specifies legume cortical cell fate to enable nodulation. Nature.

Dénarié J, Debellé F, Promé JC. 1996. Rhizobium lipo-chitooligosaccharide nodulation factors: signaling molecules mediating recognition and morphogenesis. Annu Rev Biochem 65, 503-535. El Yahyaoui F, Kuster H, Ben Amor B, Hohnjec N, Puhler A, Becker A, Gouzy J, Vernie T, Gough C, Niebel A, Godiard L, Gamas P. 2004. Expression profiling in Medicago truncatula identifies more than 750 genes differentially expressed during nodulation, including many potential regulators of the symbiotic program. Plant Physiol 136, 3159-3176.

Esseling JJ, Lhuissier FG, Emons AM. 2003. Nod factor-induced root hair curling: continuous polar growth towards the point of nod factor application. Plant Physiol 132, 1982-1988.

Fariello MI, Boitard S, Mercier S, Robelin D, Faraut T, Arnould C, Recoquillay J, Bouchez O, Salin G, Dehais P, Gourichon D, Leroux S, Pitel F, Leterrier C, SanCristobal M. 2017. Accounting for linkage disequilibrium in genome scans for selection without individual genotypes: The local score approach. Mol Ecol 26, 3700-3714.

Feng F, Sun J, Radhakrishnan GV, Lee T, Bozsóki Z, Fort S, Gavrin A, Gysel K, Thygesen MB, Andersen KR, Radutoiu S, Stougaard J, Oldroyd GED. 2019. A combination of chitooligosaccharide and lipochitooligosaccharide recognition promotes arbuscular mycorrhizal associations in Medicago truncatula. Nat Commun 10, 5047.

Fonouni-Farde C, Miassod A, Laffont C, Morin H, Bendahmane A, Diet A, Frugier F. 2019. Gibberellins negatively regulate the development of Medicago truncatula root system. Sci Rep $\mathbf{9}$, 2335.

Foo E, Ross JJ, Jones WT, Reid JB. 2013. Plant hormones in arbuscular mycorrhizal symbioses: an emerging role for gibberellins. Ann Bot 111, 769-779.

Genre A, Chabaud M, Balzergue C, Puech-Pagès V, Novero M, Rey T, Fournier J, Rochange S, Bécard G, Bonfante P, Barker DG. 2013. Short-chain chitin oligomers from arbuscular mycorrhizal fungi trigger nuclear $\mathrm{Ca} 2+$ spiking in Medicago truncatula roots and their production is enhanced by strigolactone. New Phytol 198, 190-202.

Girardin A, Wang T, Ding Y, Keller J, Buendia L, Gaston M, Ribeyre C, Gasciolli V, Auriac M-C, Vernie $T$, Bendahmane A, Ried MK, Parniske M, Morel P, Vandenbussche M, Schorderet M, Reinhardt D, Delaux P-M, Bono J-J, Lefebvre B. 2019. LCO Receptors Involved in Arbuscular Mycorrhiza Are Functional for Rhizobia Perception in Legumes. Current Biology 29, 4249-+.

Gough C, Cullimore J. 2011. Lipo-chitooligosaccharide signaling in endosymbiotic plant-microbe interactions. Mol Plant Microbe Interact 24, 867-878.

Grillo MA, De Mita S, Burke PV, Solórzano-Lowell KL, Heath KD. 2016. Intrapopulation genomics in a model mutualist: Population structure and candidate symbiosis genes under selection in Medicago truncatula. Evolution 70, 2704-2717.

Haruta M, Sabat G, Stecker K, Minkoff BB, Sussman MR. 2014. A peptide hormone and its receptor protein kinase regulate plant cell expansion. Science 343, 408-411.

Hattori J, Boutilier KA, van Lookeren Campagne MM, Miki BL. 1998. A conserved BURP domain defines a novel group of plant proteins with unusual primary structures. Mol Gen Genet 259, 424428.

Helariutta Y, Fukaki H, Wysocka-Diller J, Nakajima K, Jung J, Sena G, Hauser MT, Benfey PN. 2000. The SHORT-ROOT gene controls radial patterning of the Arabidopsis root through radial signaling. Cell 101, 555-567.

Herrbach V, Chirinos X, Rengel D, Agbevenou K, Vincent R, Pateyron S, Huguet S, Balzergue S, Pasha A, Provart N, Gough C, Bensmihen S. 2017. Nod factors potentiate auxin signaling for transcriptional regulation and lateral root formation in Medicago truncatula. J Exp Bot 68, 569-583. 
Hogekamp C, Arndt D, Pereira PA, Becker JD, Hohnjec N, Küster H. 2011. Laser microdissection unravels cell-type-specific transcription in arbuscular mycorrhizal roots, including CAAT-box transcription factor gene expression correlating with fungal contact and spread. Plant Physiol 157, 2023-2043.

Hu B, Wu H, Huang W, Song J, Zhou Y, Lin Y. 2019. SWEET Gene Family in Medicago truncatula: Genome Wide identification, expression and substrate specificity analysis. Plants (Basel) 8.

Jardinaud MF, Boivin S, Rodde N, Catrice O, Kisiala A, Lepage A, Moreau S, Roux B, Cottret L, Sallet E, Brault M, Emery RJ, Gouzy J, Frugier F, Gamas P. 2016. A laser dissection-RNAseq analysis highlights the activation of cytokinin pathways by Nod factors in the Medicago truncatula root epidermis. Plant Physiol.

Kang HM, Sul JH, Service SK, Zaitlen NA, Kong SY, Freimer NB, Sabatti C, Eskin E. 2010. Variance component model to account for sample structure in genome-wide association studies. Nat Genet 42, 348-354.

Kang Y, Sakiroglu M, Krom N, Stanton-Geddes J, Wang M, Lee YC, Young ND, Udvardi M. 2015. Genome-wide association of drought-related and biomass traits with HapMap SNPs in Medicago truncatula. Plant Cell Environ.

Kryvoruchko IS, Sinharoy S, Torres-Jerez I, Sosso D, Pislariu Cl, Guan D, Murray J, Benedito VA, Frommer WB, Udvardi MK. 2016. MtSWEET11, a Nodule-Specific Sucrose Transporter of Medicago truncatula. Plant Physiol 171, 554-565.

Liang Y, Cao Y, Tanaka K, Thibivilliers S, Wan J, Choi J, Kang C, Qiu J, Stacey G. 2013. Nonlegumes respond to rhizobial Nod factors by suppressing the innate immune response. Science 341, 13841387.

Liang Y, Tóth K, Cao Y, Tanaka K, Espinoza C, Stacey G. 2014. Lipochitooligosaccharide recognition: an ancient story. New Phytol 204, 289-296.

Limpens E, van Zeijl A, Geurts R. 2015. Lipochitooligosaccharides modulate plant host immunity to enable endosymbioses. Annu Rev Phytopathol 53, 311-334.

Maillet F, Poinsot V, André O, Puech-Pages V, Haouy A, Gueunier M, Cromer L, Giraudet D, Formey D, Niebel A, Martinez EA, Driguez H, Bécard G, Dénarié J. 2011. Fungal lipochitooligosaccharide symbiotic signals in arbuscular mycorrhiza. Nature 469, 58-63.

Mashiguchi K, Seto Y, Yamaguchi S. 2020. Strigolactone biosynthesis, transport and perception. Plant J.

Nakagawa T, Kaku H, Shimoda Y, Sugiyama A, Shimamura M, Takanashi K, Yazaki K, Aoki T, Shibuya $\mathbf{N}$, Kouchi H. 2011. From defense to symbiosis: limited alterations in the kinase domain of LysM receptor-like kinases are crucial for evolution of legume-Rhizobium symbiosis. Plant Journal 65. Ohsten Rasmussen M, Hogg B, Bono JJ, Samain E, Driguez H. 2004. New access to lipochitooligosaccharide nodulation factors. Org Biomol Chem 2, 1908-1910.

Olah B, Briere C, Bécard G, Dénarié J, Gough C. 2005. Nod factors and a diffusible factor from arbuscular mycorrhizal fungi stimulate lateral root formation in Medicago truncatula via the DMI1/DMI2 signalling pathway. Plant J 44, 195-207.

Pecrix Y, Staton SE, Sallet E, Lelandais-Brere C, Moreau S, Carrere S, Blein T, Jardinaud MF, Latrasse D, Zouine M, Zahm M, Kreplak J, Mayjonade B, Satge C, Perez M, Cauet S, Marande W, ChantryDarmon C, Lopez-Roques C, Bouchez O, Berard A, Debelle F, Munos S, Bendahmane A, Berges H, Niebel A, Buitink J, Frugier F, Benhamed M, Crespi M, Gouzy J, Gamas P. 2018. Whole-genome landscape of Medicago truncatula symbiotic genes. Nature Plants 4, 1017-1025.

Radutoiu S, Madsen LH, Madsen EB, Jurkiewicz A, Fukai E, Quistgaard EM, Albrektsen AS, James EK, Thirup S, Stougaard J. 2007. LysM domains mediate lipochitin-oligosaccharide recognition and Nfr genes extend the symbiotic host range. Embo J 26, 3923-3935.

Rao S, Zhou Z, Miao P, Bi G, Hu M, Wu Y, Feng F, Zhang X, Zhou JM. 2018. Roles of Receptor-Like Cytoplasmic Kinase VII Members in Pattern-Triggered Immune Signaling. Plant Physiol 177, 16791690. 
Rey T, Andre O, Nars A, Dumas B, Gough C, Bottin A, Jacquet C. 2019. Lipo-chitooligosaccharide signalling blocks a rapid pathogen-induced ROS burst without impeding immunity. New Phytologist 221, 743-749.

Rey T, Bonhomme M, Chatterjee A, Gavrin A, Toulotte J, Yang W, André O, Jacquet C, Schornack S. 2017. The Medicago truncatula GRAS protein RAD1 supports arbuscular mycorrhiza symbiosis and Phytophthora palmivora susceptibility. J Exp Bot 68, 5871-5881.

Ristova D, Giovannetti M, Metesch K, Busch W. 2018. Natural genetic variation shapes root system responses to phytohormones in Arabidopsis. Plant J 96, 468-481.

Roche $P$, Debellé F, Maillet F, Lerouge P, Faucher C, Truchet G, Dénarié J, Promé JC. 1991a.

Molecular basis of symbiotic host specificity in Rhizobium meliloti: nodH and nodPQ genes encode the sulfation of lipo-oligosaccharide signals. Cell 67, 1131-1143.

Roche P, Lerouge P, Ponthus C, Prome JC. 1991b. Structural determination of bacterial nodulation factors involved in the Rhizobium meliloti-alfalfa symbiosis. J Biol Chem 266, 10933-10940.

Ronfort J, Bataillon T, Santoni S, Delalande M, David JL, Prosperi JM. 2006. Microsatellite diversity and broad scale geographic structure in a model legume: building a set of nested core collection for studying naturally occurring variation in Medicago truncatula. BMC Plant Biol 6, 28.

Rush T, Puech-Pagès V, Bascaules A, Jargeat $P$, Maillet $F$, Haouy A, Maës A, Carrera Carriel $C$, Khokhani D, Keller-Pearson M, Tannous J, Cope K, Garcia K, Maeda J, Johnson C, Kleven B, Choudhury Q, Labbé, J., Swift C, O'Malley M, Bok J, Cottaz S, Fort S, Poinsot V, Sussman M, Lefort C, Nett J, Keller N, Bécard G, Ané J. 2020. Lipo-chitooligosaccharides as regulatory signals of fungal growth and development. Nature Commun in press.

Samain E, Chazalet V, Geremia RA. 1999. Production of O-acetylated and sulfated chitooligosaccharides by recombinant Escherichia coli strains harboring different combinations of nod genes. J Biotechnol 72, 33-47.

Samain E, Drouillard S, Heyraud A, Driguez H, Geremia RA. 1997. Gram-scale synthesis of recombinant chitooligosaccharides in Escherichia coli. Carbohydr Res 302, 35-42.

Sassi M, Lu Y, Zhang Y, Wang J, Dhonukshe P, Blilou I, Dai M, Li J, Gong X, Jaillais Y, Yu X, Traas J, Ruberti I, Wang H, Scheres B, Vernoux T, Xu J. 2012. COP1 mediates the coordination of root and shoot growth by light through modulation of PIN1- and PIN2-dependent auxin transport in Arabidopsis. Development 139, 3402-3412.

Sauer M, Kleine-Vehn J. 2019. PIN-FORMED and PIN-LIKES auxin transport facilitators. Development 146.

SchiessI K, Lilley JLS, Lee T, Tamvakis I, Kohlen W, Bailey PC, Thomas A, Luptak J, Ramakrishnan K, Carpenter MD, Mysore KS, Wen J, Ahnert S, Grieneisen VA, Oldroyd GED. 2019. NODULE INCEPTION Recruits the Lateral Root Developmental Program for Symbiotic Nodule Organogenesis in Medicago truncatula. Curr Biol.

Souleimanov A, Prithiviraj B, Smith DL. 2002. The major Nod factor of Bradyrhizobium japonicum promotes early growth of soybean and corn. J Exp Bot 53, 1929-1934.

Soyano T, Shimoda Y, Kawaguchi M, Hayashi M. 2019. A shared gene drives lateral root development and root nodule symbiosis pathways in Lotus. Science 366, 1021-1023.

Sparks E, Wachsman G, Benfey PN. 2013. Spatiotemporal signalling in plant development. Nat Rev Genet 14, 631-644.

Stanton-Geddes J, Paape T, Epstein B, Briskine R, Yoder J, Mudge J, Bharti AK, Farmer AD, Zhou P, Denny R, May GD, Erlandson S, Yakub M, Sugawara M, Sadowsky MJ, Young ND, Tiffin P. 2013. Candidate genes and genetic architecture of symbiotic and agronomic traits revealed by wholegenome, sequence-based association genetics in Medicago truncatula. PLoS One 8, e65688.

Stegmann M, Monaghan J, Smakowska-Luzan E, Rovenich H, Lehner A, Holton N, Belkhadir Y, Zipfel C. 2017. The receptor kinase FER is a RALF-regulated scaffold controlling plant immune signaling. Science 355, 287-289.

Sulima AS, Zhukov VA, Afonin AA, Zhernakov AI, Tikhonovich IA, Lutova LA. 2017. Selection Signatures in the First Exon of Paralogous Receptor Kinase Genes from the Sym2 Region of the Pisum sativum L. Genome. Frontiers in Plant Science 8. 
Sulima AS, Zhukov VA, Kulaeva OA, Vasileva EN, Borisov AY, Tikhonovich IA. 2019. New sources of Sym $2{ }^{A}$ allele in the pea ( Pisum sativum L.) carry the unique variant of candidate LysM-RLK gene LykX. PeerJ 7, e8070.

Sun J, Miller JB, Granqvist E, Wiley-Kalil A, Gobbato E, Maillet F, Cottaz S, Samain E, Venkateshwaran M, Fort S, Morris RJ, Ané JM, Dénarié J, Oldroyd GE. 2015. Activation of symbiosis signaling by arbuscular mycorrhizal fungi in legumes and rice. Plant Cell 27, 823-838.

Tanaka K, Cho SH, Lee H, Pham AQ, Batek JM, Cui S, Qiu J, Khan SM, Joshi T, Zhang ZJ, Xu D, Stacey G. 2015. Effect of lipo-chitooligosaccharide on early growth of C4 grass seedlings. J Exp Bot 66, 57275738.

Trujillo DI, Silverstein KA, Young ND. 2014. Genomic characterization of the LEED..PEEDs, a gene family unique to the medicago lineage. G3 (Bethesda) 4, 2003-2012.

Wang D, Couderc F, Tian CF, Gu W, Liu LX, Poinsot V. 2018. Conserved Composition of Nod Factors and Exopolysaccharides Produced by Different Phylogenetic Lineage. Front Microbiol 9, 2852.

Wang L, Wu N, Zhu Y, Song W, Zhao X, Li Y, Hu Y. 2015. The divergence and positive selection of the plant-specific BURP-containing protein family. Ecol Evol 5, 5394-5412.

Yamaguchi S. 2008. Gibberellin metabolism and its regulation. Annu Rev Plant Biol 59, 225-251. Yoder JB, Stanton-Geddes J, Zhou P, Briskine R, Young ND, Tiffin P. 2014. Genomic signature of adaptation to climate in Medicago truncatula. Genetics 196, 1263-1275.

Table 1 - Estimation of narrow-sense heritability for different phenotypic variables measuring lateral root stimulation.

\begin{tabular}{|c|c|c|c|c|c|}
\hline & \multirow{2}{*}{$\begin{array}{l}\text { Days post } \\
\text { treatment }\end{array}$} & \multicolumn{2}{|c|}{ Fung-LCO treatment } & \multicolumn{2}{|c|}{ Nod-LCO treatment } \\
\hline & & Heritability & $\begin{array}{l}\% \text { accessions with } \\
\Delta>0 \text { (stimulation) }\end{array}$ & Heritability & $\begin{array}{l}\% \text { accessions with } \\
\Delta>0 \text { (stimulation) }\end{array}$ \\
\hline$\Delta$ _lateral_root_number & 5 & 0 & $71.7(++)$ & 0.66 & $82.7(++)$ \\
\hline$\Delta$ _lateral_root_number & 8 & 0.03 & $75.7(++)$ & 0.48 & $90.2(+++)$ \\
\hline$\Delta$ _lateral_root_number & 11 & 0.11 & $75.1(++)$ & 0.22 & $82.1(++)$ \\
\hline$\Delta$ _lateral_root_number & 15 & 0.16 & 47.3 & 0.35 & $77.5(++)$ \\
\hline$\Delta \_$AULRPC & $\begin{array}{l}\text { 5-8-11-15 } \\
\text { (kinetics) }\end{array}$ & 0.12 & $67.2(+)$ & 0.50 & $86.7(+++)$ \\
\hline$\Delta$ _lateral_root_density & 5 & 0.06 & $66.5(+)$ & 0.75 & $68.8(+)$ \\
\hline$\Delta$ _lateral_root_density & 11 & 0.15 & $64.7(+)$ & 0.36 & $81.5(++)$ \\
\hline $\Delta$ _primary_root_length & 5 & 0.14 & $92.5(+++)$ & 0.22 & $56.6(+)$ \\
\hline$\Delta$ A_primary_root_length & 11 & 0 & $82.1(++)$ & 0.36 & $69.9(+)$ \\
\hline
\end{tabular}

$+: 55<\%_{\Delta>0}<70,++: 70<\%_{\Delta>0}<85,+++: \%_{\Delta>0}>85$. 

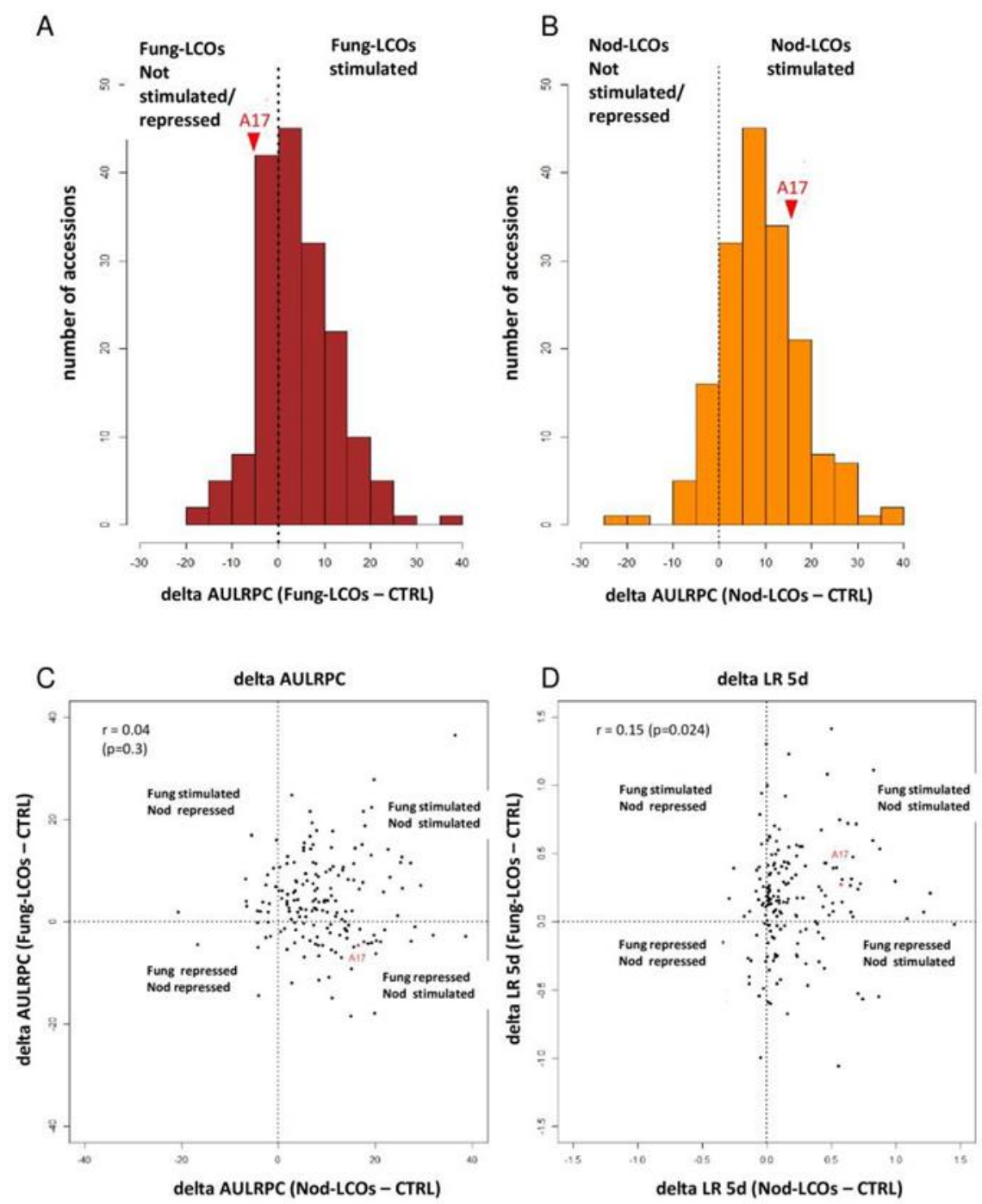

Figure 1-Medicago truncatula stimulation of root development by Fung- and Nod-LCOs

Quantitative variation in the stimulation of root development is observed in response to (A) Fung- and (B) Nod-LCOs, with $67 \%$ and $87 \%$ of the 173 accessions of $M$. truncatula showing stimulation of root development, respectively. This root development was measured for 15 days and expressed as the delta_AULRPC (see Fig. S2). The position of the reference genotype A17, 
relative to the other accessions, is indicated by a red arrow head. (C) Plot of delta_AULRPC (Nod-LCOs - CTRL) values versus delta_AULRPC (Fung-LCOs - CTRL) values and (D) plot of delta_LR_5d (Nod-LCOs - CTRL) versus delta_LR_5d (Fung-LCOs - CTRL) values for 173 accessions of Medicago truncatula, indicating a weak correlation between the stimulation by Fung- and Nod-LCOs. Vertical and horizontal dashed lines indicate equal states of root development between treatment (Fung- or Nod-LCOs) and control conditions (CTRL). The reference genotype A17 is indicated in red and with an arrowhead.
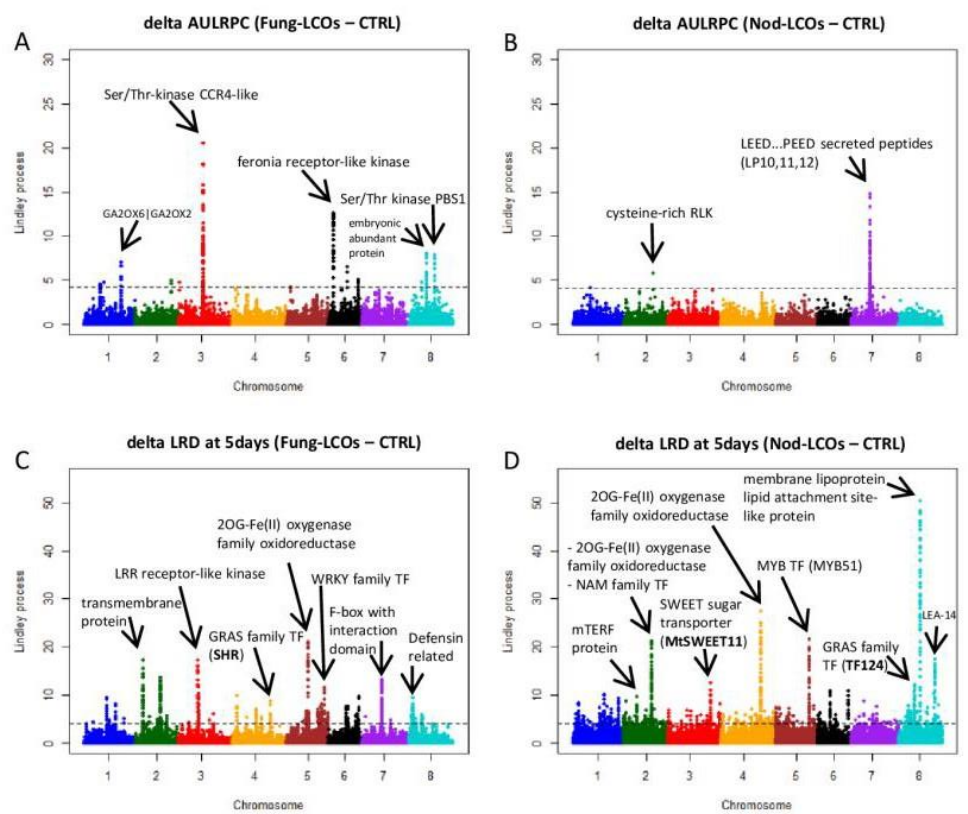

Figure 2 - GWAS results using a local score approach on Medicago truncatula stimulation of lateral root development by Fung- and Nod-LCOs.

Each Manhattan plot shows on the y-axis the Lindley process (the local score with the tuning parameter $\xi=3$ ) for SNPs along the eight chromosomes (x-axis), with the dashed line indicating the maximum of the eight chromosome-wide significance thresholds. The local score is shown for GWAS of four phenotypic variables: (A) delta_AULRPC (Fung-LCOs - CTRL), (B) delta_AULRPC (Nod-LCOs - CTRL), (C) delta_LRD_5d (Fung-LCOs - CTRL) and (D) delta_LRD_5d (Nod-LCOs - CTRL). The most significant candidate genes and their predicted functions are indicated by arrows on the plots (see Table S5). 
A
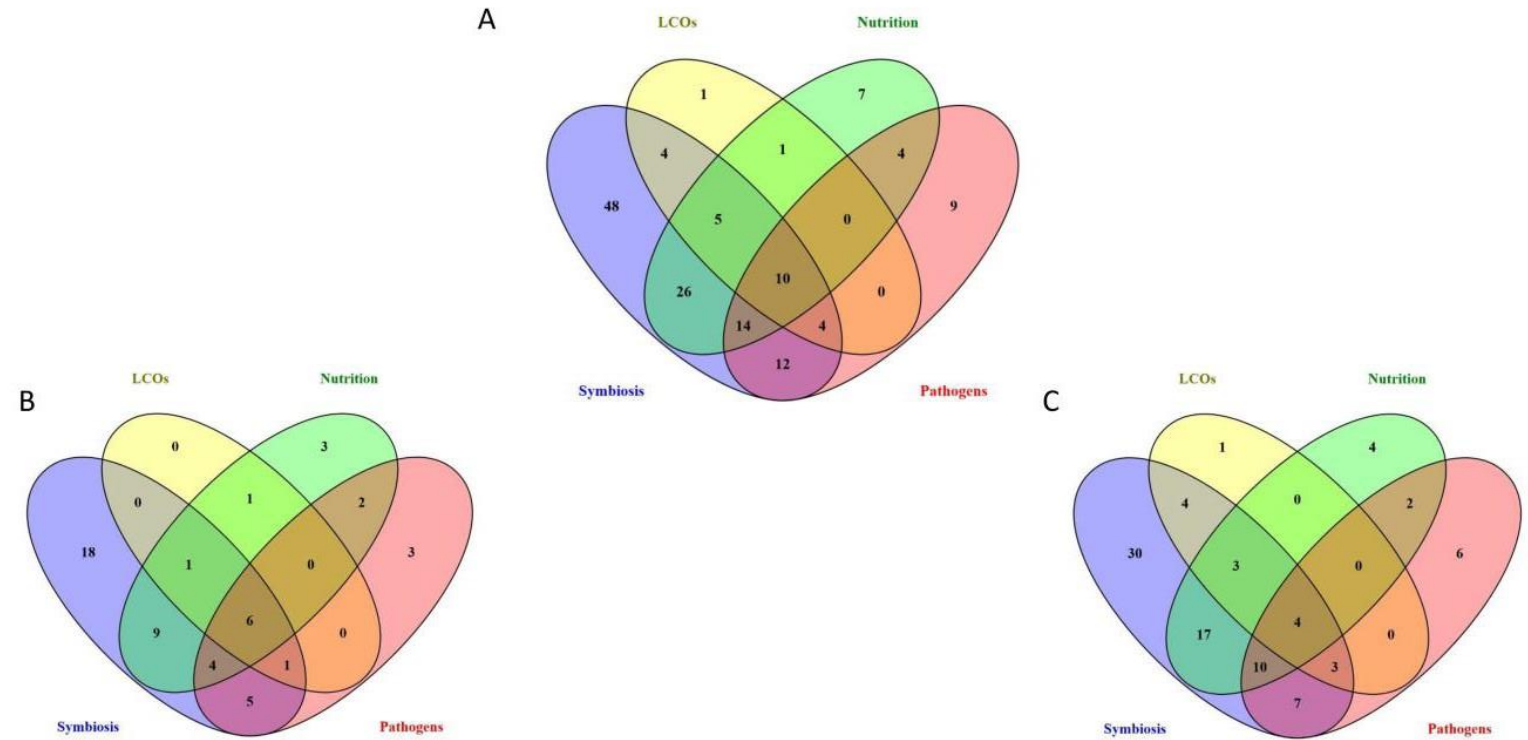

Figure 3. Venn diagrams of genes found as differentially expressed in transcriptomic data of various biological conditions from the literature.

(A) 148 GWAS candidate loci from both GWAS analyses corresponding to (B) 54 Fung-LCOs or (C) 93 Nod-LCOs loci were found as differentially expressed genes from the literature in the LEGOO knowledge database (Carrère et al., 2020): Symbiosis (blue circle, inoculation with $S$. meliloti or R. irregularis), Pathogens (red circle, Aphanomyces euteiches, Phymatotrichopsis omnivora, Ralstonia solanacearum), nutrition (green circle, phosphate and nitrate starvation) or LCOs (yellow circle, S. meliloti Nod Factors, sulfated or unsulfated Myc-LCOs as described in Maillet et al., 2011) (see Table S6 for details). 